\title{
Shifting paradigm in brain abscess management at tertiary care centre in Nepal
}

\author{
Prakash Kafle', Mohan Raj Sharma², Sushil Krishna Shilpakar², Gopal Sedain², Amit Pradhanang², \\ Ram Kumar Shrestha ${ }^{2}$, Binod Raj Bhandari ${ }^{2}$, Christine Groves ${ }^{3}$ \\ 'Department of Neurosurgery, Nobel Medical College Teaching Hospital, Biratnagar 56613, Nepal. \\ 2Department of Neurosurgery, Tribhuvan University Teaching Hospital, Kathmandu 44613, Nepal. \\ ${ }^{3}$ Department of Physical Medicine and Rehabilitation, Indiana University School of Medicine, Indianapolis, IN 46202, USA.
}

Correspondence to: Dr. Prakash Kafle, Department of Neurosurgery, Nobel Medical College Teaching Hospital, Biratnagar 56613, Nepal. E-mail: prakashkaflee@gmail.com

How to cite this article: Kafle P, Sharma MR, Shilpakar SK, Sedain G, Pradhanang A, Shrestha RK, Bhandari BR, Groves C. Shifting paradigm in brain abscess management at tertiary care centre in Nepal. Neuroimmuno/ Neuroinflammation 2018;5:24. https://doi.org/10.20517/2347-8659.2018.10

Received: 21 Mar 2018 First Decision: 28 Apr 2018 Revised: 16 May 2018 Accepted: 17 May 2018 Published: 7 Jun 2018

Science Editor: Athanassios P. Kyritsis Copy Editor: Jun-Yao Li Production Editor: Cai-Hong Wang

\begin{abstract}
Aim: Brain abscess is a challenging clinical entity with substantial high case fatality rates despite significant advances in imaging techniques, laboratory modalities, surgical interventions, and antimicrobial treatment.Otogenic and cardiogenic sources are among the most common. Classic clinical presentation is seen in very few cases only. Burr hole with aspiration works well with good clinical outcomes. Control of primary source in cases of ear infection in the single setting results in good outcomes, reduces for additional surgery, and decreases the duration of hospital stay.
\end{abstract}

Methods: This is prospective observational study conducted at Institute of Medicine, Tribhuvan University Teaching Hospital, Kathmandu, Nepal over the period of two and a half years (from September 2014 to March 2017).We analyzed the demographic profile, management strategies and outcome of these cases diagnosed with brain abscess using Microsoft Excel 2007.

Results: A total of 51 cases were undertaken for surgical management. There were 35 males and 16 females with the male to female ratio of 2.18:1. The mean age of the study population was 16.76 years with age range from 4 months to 60 years. Otogenic source was the most common. Temporal lobe was the most common abscess location. Headache was the most common clinical presentation and was seen in $86.27 \%$ of the study population. All cases were initially managed with burrhole and aspiration of the abscess. Only 3.92\% $(n=2)$ of cases subsequently required surgical excision of the abscess wall. Only $11.76 \%(n=6)$ of the cases required multiple aspiration. Only $19.61 \%(n=10)$ showed positive culture. Pseudomonas aeruginosa and E. coli were the most common organisms grown. Mortality rate among the study group was $3.92 \%$.




Conclusion: With the advent of modern technology in neuroimaging, mortality due to brain abscess has significantly decreased. Joint involvement of the otorhinolaryngology team and efforts in addressing the primary source have further helped in improving outcomes in cases of otogenic brain abscess. Hence, source control is of paramount importance in managing the brain abscess.

Keywords: Abscess, otogenic brain abscess, tubercular abscess

\section{INTRODUCTION}

Though the incidence of brain abscesses is as low as $2 \%$ in the western world, it accounts for up to $8 \%$ of intracranial masses in developing countries ${ }^{[1,2]}$. There is a paucity of data about the exact incidence of brain abscess in Nepal. Despite recent advances in imaging techniques, laboratory modalities, surgical interventions and antimicrobial treatment, brain abscess still remains a challenging clinical entity with substantially high case fatality rates. This is attributable to changes in epidemiology, clinical spectrum, predisposing factors and the prevalence of implicated bacterial pathogens. Brain abscess contributes to high mortality, and even more so in immune compromised patients. Different organisms have been implicated in its etiology comprising of bacteria, mycobacterium, fungi, parasites (protozoa and helminthes) and cryptogenic ${ }^{[3]}$. Brain abscess formation may occur after neurosurgical procedures or head trauma. Brain abscesses due to contiguous spread from parameningeal foci of infection (e.g., the middle ears, mastoids, and sinuses) are common in developing countries. Many of the patients with brain abscess have concomitant ear infection and/or cardiac problems, especially cyanotic heart disease. Formation of brain abscess after penetrating head injuries and following cranial surgery is also common. The present study presents the demographic profile, surgical management, microbiology and outcomes at our centre.

\section{METHODS}

This is a prospective observational study conducted over the period of two and half years (from September 2014 to March 2017) at Institute of Medicine (IOM), Tribhuvan University Teaching Hospital Kathmandu, Nepal. Data regarding patient characteristics, clinical profile, etiology, microbiology profile, management algorithm and complications were collected and analyzed. All cases with brain abscess admitted to IOM were included in the study. Individuals with brain abscess operatively managed in other hospitals and referred to our centre postoperatively were excluded from the study. Clinical findings at presentation were noted and subsequently contrast enhanced computed tomography (CECT) was done in all cases. High resolution computed tomography (HRCT) of the involved ear was also performed in indicated cases. Initially, hyperosmolar therapy (mannitol-1 gm/kg/ IV dose q 8 hourly and dexamethasone 1 - $4 \mathrm{mg}$ IV q 8 hourly depending up on the weight of patient) was instituted until surgical intervention was completed and steroids were tapered. Antiepileptic medications were instituted for all supratentorial abscesses for a minimum of 1 month and continued in patients with seizures for a period of 2 years. Burr hole and drainage is the standard procedure performed in our centre after marking the operative site on CT scan. Craniotomy and abscess cavity excision were required in very few cases. After drainage, obtained samples were sent for Gram stain, culture and sensitivity. Anaerobic culture was not done due to resource constraints. Patients were treated with initial empirical triple broad spectrum antibiotics which included vancomycin $15 \mathrm{mg} / \mathrm{kg}$ IV 8 hourly, ceftriaxone $25 \mathrm{mg} / \mathrm{kg}$ IV in two dived doses and metronidazole $15 \mathrm{mg} / \mathrm{kg} \mathrm{IV}$ in 3 divided doses. Narrowing of antibiotics was made accordingly and continued for 6 weeks if culture sensitivity was positive for a specific organism. Follow-up head CT was performed in 1 week and every 2 weeks thereafter. Head CT was performed in between if indicated by alteration in clinical status of the patient, i.e. drop in Glasgow Coma Scale by $\geq 2$ points. Repeat aspiration was performed if the size of the abscess was found to be increasing or clinical status was not improving. Patients were labeled as cured radiologically if there was no residual abscess cavity on CECT after 6 weeks of treatment. 
Table 1. Etiology of brain abscess in the study population

\begin{tabular}{llcc}
\hline No. & Etiology/source & Frequency (n) & Percentage (\%) \\
\hline 1 & Otogenic (chronic suppurative otitis media) & 28 & 54.88 \\
2 & Tubercular & 7 & 13.72 \\
3 & Cardiogenic & 6 & 11.76 \\
4 & Animal bite & 2 & 3.92 \\
6 & Post craniotomy & 1 & 1.96 \\
7 & Unknown & 7 & 13.72 \\
\hline
\end{tabular}

Table 2. Location of the intracranial abscess in the study population

\begin{tabular}{lcc}
\hline Intracranial suppuration & Frequency $(\boldsymbol{n})$ & Percentage (\%) \\
\hline Temporal abscess & 22 & 43.12 \\
Cerebellar abscess & 8 & 15.68 \\
Frontal abscess & 7 & 13.72 \\
Multiple abscess & 6 & 11.76 \\
Parietal abscess & 4 & 7.84 \\
Subdural empyema & 2 & 3.92 \\
Epidural abscess & 1 & 1.96 \\
Interhemispheric abscess & 1 & 1.96 \\
Total & 51 & 100 \\
\hline
\end{tabular}

Table 3. Dominant clinical presentation of study population

\begin{tabular}{|c|c|c|c|c|}
\hline No. & Clinical presentation & Average duration & Frequency $(n)$ & Percentage (\%) \\
\hline 1 & Headache & 30 days & 44 & 86.24 \\
\hline 2 & Ear discharge/ache & $>1$ year & 28 & 54.88 \\
\hline 3 & Focal neurological deficits & 7 days & 11 & 21.56 \\
\hline 4 & Vomiting & 4 days & 8 & 15.68 \\
\hline 5 & Altered sensorium & 2 days & 5 & 9.80 \\
\hline 6 & Seizure & 1 day & 5 & 9.80 \\
\hline 7 & Fever & 7 days & 5 & 9.80 \\
\hline
\end{tabular}

\section{RESULTS}

In the present study, 51 cases were undertaken for surgical management. There were 35 males and 16 females with the male to female ratio of 2.18:1. Mean age of the study population was 16.76 years, ranging from 4 months to 60 years. More than $50 \%$ of the study population $(n=26)$ were $\leq 16$ years old. Abscesses were primarily otogenic in origin [Table 1]. There were 28 cases of otogenic origin as a result of chronic suppurative otitis media (CSOM), followed by other identified sources including tubercular abscess $(n=7)$, cardiogenic $(n=6)$. In $13.72 \%(n=7)$ cases, the cause of brain abscess was not identified. Supratentorial lesion $(n=43)$ was more common than infratentorial $(n=8)$, and the temporal lobe was the most common site (43.13\%) [Table 2].

\section{Clinical presentation}

Headache and ear discharge were the most common presenting features [Table 3]. Almost all patients who could communicate were complaining of some degree of headache. Of those patients with CSOM $(n=44)$, $86.27 \%$ had history of ear discharge. Focal neurological deficits were seen in $29.41 \%(n=15)$ of the study population. Vomiting was seen in $29.41 \%$ of the cases $(n=15) ; 9.8 \%(n=5)$ of the study population presented to the emergency department in a state of altered sensorium and 5 patients presented with seizure. Two infants presented with poor feeding. One infant was brought in with history of infected left frontal scalp wound and was found to have exposed brain parenchyma with subdural empyema.

\section{Radiological evaluation}

CT with contrast was the initial diagnostic modality in clinically suspected cases of brain abscess. Magnetic resonance imaging (MRI) was done in cases with subsequent diagnostic uncertainty only. The most common 




Figure 1. Contrast enhanced computed tomography of head showing left temporal lobe abscess

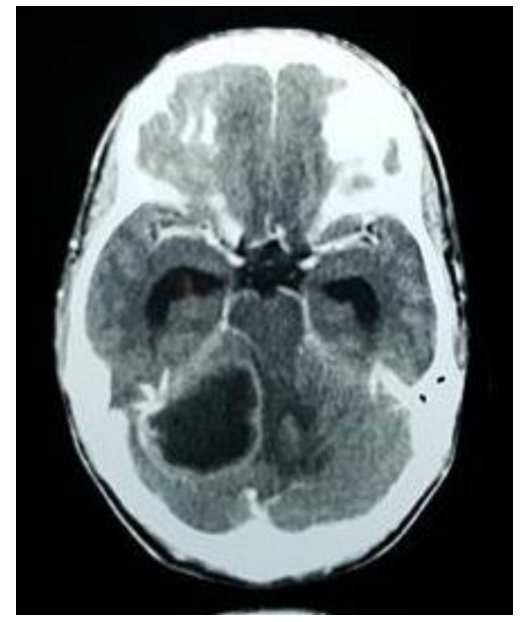

Figure 2. Contrast enhanced computed tomography of case diagnosed with brain posterior fosa (right cerebellar) abscess with chronic suppurative otitis media

CT findings showed smooth, round/oval ring enhancing lesion with central hypo density and surrounding hypo density suggestive of edema. Peripheral enhancing lesions in the subdural and epidural spaces with or without air density were suggestive of subdural empyema and epidural abscess. Destruction of the middle ear and soft tissue density in the mastoid air cells suggested suppurative otitis media which is shown in Figures 1 and 2.

\section{Management}

All cases were managed with burrhole and aspiration of the abscess initially. Only 3.92\% $(n=2)$ of cases required additional surgical excision of the abscess wall. Primary control of the source was done by modified radical mastoidectomy (MRM) in patients with $\operatorname{CSOM}(n=23)$. In 5 cases of CSOM, concomitant MRM was not done at the time of abscess drainage due to poor clinical condition. These cases subsequently underwent MRM as soon as possible, and re-aspiration was done if indicated at that time. Only $11.76 \%(n=6)$ of the cases required multiple aspiration (2-5 times) through the same burrhole [Table 4].

\section{Microbiology}

Most of the specimens sent for culture and sensitivity were negative for growth, presumably due to the use of preoperative antibiotics. Only $19.61 \%(n=10)$ showed positive culture. Pseudomonas aeruginosa and E. coli were isolated in 7 and 2 cases respectively. One case showed growth of multiple organisms including 
Table 4. Surgical procedures in the present study

\begin{tabular}{lcc}
\hline Procedure & Frequency $(\boldsymbol{n})$ & Percentage (\%) \\
\hline Burrhole and aspiration with modified radical mastoidectomy & 23 & 45.08 \\
Burrhole and aspiration & 14 & 27.44 \\
Burrhole and multiple aspiration & 6 & 11.76 \\
Craniotomy and subdural empyema drainage & 3 & 5.88 \\
Craniotomy and abscess wall excision & 2 & 3.92 \\
Continuous abscess drainage & 2 & 3.92 \\
Craniotomy and epidural abscess drainage & 1 & 1.96 \\
Total & 51 & 100 \\
\hline
\end{tabular}

Table 5. Complications observed in the study population

\begin{tabular}{llcc}
\hline No. & Complications & Frequency $(\boldsymbol{n})$ & Percentage (\%) \\
\hline 1 & Mortality & 2 & 3.92 \\
2 & Pyoventricle & 1 & 1.96 \\
3 & Post modified radical mastoidectomy facial nerve palsy (grade II-V) & 4 & 7.84 \\
4 & Pseudomeningocele & 1 & 1.96 \\
5 & Surgical site infection & 1 & 1.96 \\
\hline
\end{tabular}

staphylococcus aureus. All cases were treated with 2 weeks of intravenous and 4 weeks of oral antibiotics (Cefpodoxime $5 \mathrm{mg} / \mathrm{kg} 12$ hourly and Metronidazole $25 \mathrm{mg} / \mathrm{kg} /$ day in 3 divided doses) including anaerobic coverage. Some cases required 8 weeks of antibiotics. Oral versus intravenous route was determined depending on the status of the abscess cavity on repeat head CT. Culture and sensitivity for anaerobic organisms were not done in the present study due to resource constrains.

\section{Complications}

The mortality was 3.92\% $(n=2)$ during the study. Major complications observed are listed in Table 5. The most common minor complication noted was thrombophlebitis likely due to prolonged use of IV antibiotics.

\section{Outcome}

All surviving patients were followed up in outpatient clinic for at least 3 months. All of them had Glasgow outcome scale of 5/5. Surgical site infection and pseudomeningocele was resolved at the 6 week follow up visit. Facial palsy resolved in 3 cases, with residual palsy present in 1 case at follow-up cessation (3 months).

\section{DISCUSSION}

Brain abscess comprises approximately $8 \%$ of all space occupying lesions in the brain in developing countries ${ }^{[1,2]}$. Abscess is the second most common type of intracranial complication of otogenic origin, with temporal lobe being the most common site of pathology. Clinical presentation varies among patients. The classic triad of fever, headache and focal deficit is rarely seen. Features of raised intracranial pressure with or without localizing signs require early radiological imaging to avoid inadvertent delay in management ${ }^{[4]}$. Contrast enhanced CT scan of the head is the mainstay of diagnostic modalities ${ }^{[5]}$, providing rapid means of detecting the lesion. MRI, combined with diffusion-weighted (DWI) and apparent-diffusion coefficient (ADC) images, is a valuable diagnostic tool in differentiating brain abscess from primary, cystic, or necrotic tumors with positive predictive value of $98 \%$ and negative predictive value of $92 \%{ }^{[6]}$. Cultures of blood and cerebrospinal fluid identify the causative pathogen in approximately one quarter of patients. Cultures of cerebrospinal fluid may be valuable in patients with coexisting meningitis. Lumbar puncture can lead to herniation in such situations ${ }^{[7]}$.

There is no pragmatic rule for the treatment of brain abscess. Treatment of each case is individualized depending up on the location, size, and stage of abscess. The mainstay of treatment is prompt action and 
initiation of antibiotics. However, surgery is imperative for the identification of the causative pathogen and for the purpose of reducing the size of the abscess. With the use of modern stereotactic neurosurgical techniques, almost any brain abscess that measures at least $1 \mathrm{~cm}$ in diameter is amenable to stereotactic aspiration, regardless of location. Stereotactic navigation systems can be used for abscess drainage ${ }^{[8]}$. Nonoperative management of small abscess with broad spectrum antibiotics is also common. In the present study we managed the cases with burr hole and abscess aspiration. Abscess wall excision was done in cases which required additional procedures, like evacuation of subdural or epidural pathology. It was also indicated that following multiple aspirations fail to result in abscess resolution.

Another important aspect of care is managing the primary source. A number of studies have shown good results with concurrent abscess drainage and mastoidectomy in the same setting without added morbidity in cases with CSOM. They have shown low recurrence rate, though not statistically significant ${ }^{[9]}$. Other procedures being carried out include transmastoid approaches for abscess ${ }^{[10]}$.

Brain abscess is seen in 5\%-18\% cyanotic congenital heart diseases and these individuals are 10 times more prone to develop brain abscess then those without cyanotic heart disease. Tetralogy of Fallot is the most common cardiac condition associated with brain abscess. Right-to-left shunt, hypoxia, acidosis, and increased viscosity decrease the perfusion in the brain resulting in micro infarcts, which provide the milieu for organisms to form abscesses. The 2 mortalities in this study were associated with cardiogenic brain abscesses.

Important criteria for evaluating treatment are the neurologic condition of the patient and abscess size on imaging. Cranial imaging should be performed immediately if there is clinical deterioration; after 1 to 2 weeks if there is no improvement; and on a biweekly basis for up to 3 months until clinical recovery is evident ${ }^{[1]]}$. Culture positivity is low in cases of brain abscess. In the present study, organism growth is seen only in $19.6 \%$ cases. In a study done in India, only $20 \%$ culture growth was noted whereas in China only $13 \%$ showed organism growth ${ }^{[12]}$. Lack of anaerobic culture and use of antibiotics before samples are drawn may be the reasons behind the higher number of negative culture reports. However, metagenomics analysis and nucleotide sequence analysis are being used in some centers to identify the responsible organism and they have been able to identify bacteria that have never been incriminated as a cause for brain abscess ${ }^{[13]}$.

Tract hematoma, abscess cavity hematoma, extension of abscess into the ventricles, surgical site infection, meningitis, sinus thrombosis and mortality are known complications of brain abscess management. Apart from this, drug-related complications including minor rashes (potentially due to phenytoin use) are common side effects. We commonly use anti-epileptics at least for 1 month and continue if seizure is present. Cerebral hemisphere was the most common site $(84.32 \%)$ of abscess in the present study, which was similar to the previous study done by Sharma ${ }^{[14]}$ in the same institute. One patient developed hematoma adjacent to the aspiration site, which can be explained either by direct injury to the vessel during aspiration or reactive hemorrhage due to rapid decompression of the abscess. In the pre-CT era, mortality rate was about $40 \%-60 \%$. This has decreased to $6 \%-17 \%$ currently ${ }^{[15,16]}$. Preoperative GCS remains the best prognostic factor ${ }^{[16]}$.

There were two deaths during this study (3.92\%). The first mortality was a case of cyanotic congenital heart disease with brain abscess that died due to cardiac causes during hospital stay. The second mortality was also a child with large abscess with intraventricular rupture. Patients with intraventricular extension of abscess carry higher mortality rates of up to $48 \%$, even in this modern era ${ }^{[17]}$.

In conclusion, with the advent of modern technology in radio imaging, mortality rates of brain abscess have improved over the decades as compared in the past. Outcomes may be dismal if not treated early which is particularly challenging given non-specific presentation of many patients with brain abscess. Thus, a high 
index of suspicion is required in patients with features of raised ICP. Burrhole with aspiration is an excellent option for surgical management. Major craniotomy and excision should be preserved for multiloculated, recurrent, large size abscess cavities only. Culture positivity is very low, so longer broad spectrum intravenous and/or oral antibiotics help in early resolution. Involvement of the otorhinolaryngology team to address the primary source has further helped improve outcomes in cases of otogenic brain abscess.

\section{DECLARATIONS}

\section{Authors' contributions}

Collected all data: Kafle P, Sharma MR, Shilpakar SK, Sedain G

Followed all the patients and analyzed: Kafle P, Pradhanang A, Shrestha RK, Bhandari BR

Critical analysis, language editing and final approval: Groves $\mathrm{C}$

\section{Availability of data and materials}

The data presented is original and obtained in our laboratory. It is available with the authors and can be made available if required.

\section{Financial support and sponsorship}

None.

\section{Conflicts of interest}

All authors declared that there are no conflicts of interest.

\section{Ethical approval and consent to participate}

All treatment and study were performed in compliance with our institutional standard and the Declaration of Helsinki and consent was taken for all patients before treatment.

\section{Consent for publication}

Not applicable.

\section{Copyright}

(c) The Author(s) 2018.

\section{REFERENCES}

1. Sharma BS, Gupta SK, Khosla VK. Current concepts in the management of pyogenic brain abscess. Neurol India 2000;48:105-11.

2. Bhatia R, Tandon PN, Banerji AK. Brain abscess an analysis of 55 cases. Int Surg J 1973;58:565-68.

3. Helweg-Larsen J, Astradsson A, Richhall H, Erdal J, Laursen A, Brennum J. Pyogenic brain abscess, a 15 year survey. BMC Infect Dis $2012 ; 12: 332$.

4. Wanna GB, Dharamsi LM, Moss JR, Bennett ML, Thompson RC, Haynes DS. Contemporary management of intracranial complications of otitis media. Otol Neurotol 2010;131:111-7.

5. Krivopalov AA, Yanov YK, Astashchenko SV, Shcherbuk AY, Artyushkin SA, Vakhrushev SG, Piskunov IS, Piskunov VS, Tuzikov NA. Features of otogenic intracranial complications at the present stage. Vestn Khir Im I I Grek 2015;174:68-79. (in Russian)

6. Reddy JS, Mishra AM, Behari S, Husain M, Gupta V, Rastogi M, Gupta RK. The role of diffusion-weighted imaging in the differential diagnosis of intracranial cystic mass lesions: a report of 147 lesions. Surg Neurol 2006;66:246-50; discussion 250-1.

7. Jim KK, Brouwer MC, van der Ende A, van de Beek D. Cerebral abscesses in patients with bacterial meningitis. J Infect 2012;64:236-8.

8. Barlas O, Sencer A, Erkan K, Eraksoy H, Sencer S, Bayindir C. Stereotactic surgery in the management of brain abscess. Surg Neurol 1999;52:404-10.

9. Ozkaya S, Bezircioglu H, Sucu HK, Ozdemir I. Combined approachfor otogenic brain abscess. Neurol Med Chir (Tokyo) 2005;45:82-5, discussion 86.

10. Morwani KP, Jayashankar N. Single stage,transmastoid approach orotogenic intracranial abscess. J Laryngol Otol 2009;123:1216-20.

11. Shen H, Huo Z, Liu L, Lin Z. Stereotactic implantation of ommaya reservoir in the management of brain abscesses. Br J Neurosurg 2011;25:636-40. 
12. Singh D, Gupta V, Singh AK, Sinha S. Evolution of otogenic brainabscess and management protocol. Indian Pediatr 2001;38:169-73.

13. Al Masalma M, Lonjon M, Richet H, Dufour H, Roche PH, Drancourt M, Raoult D, Fournier PE. Metagenomic analysis of brain abscesses identifi es specifi cbacterial associations. Clin Infect Dis 2012;54:202-10.

14. Sharma MR. Management of pyogenic brain abscess in children: review of 50 cases. Nepal J Neurosci 2013;10:61-7.

15. Kao PT, Tseng HK, Liu CP, Su SC, Lee CM. Brain abscess: clinical analysis of 53 cases. J Microbiol Immunol Infect 2003;36:129-36.

16. Zhang C, Hu L, Wu X, Hu G, Ding X, Lu Y. A retrospective study on the aetiology, management, and outcome of brain abscess in an 11-year, single-centre study from China. BMC Infect Dis 2014;14:311.

17. Lee TH, Chang WN, Su TM, Chang HW, Lui CC, Ho JT, Wang HC, Lu CH. Clinical features and predictive factors of intraventricular rupture in patients who have bacterial brain abscesses. J Neurol Neurosurg Psychiatry 2007;78:303-9. 